\title{
Autocorrected off-axis holography of two-dimensional materials
}

\author{
Felix Kern $\odot,{ }^{1, *}$ Martin Linck, ${ }^{2}$ Daniel Wolf $\odot,{ }^{1}$ Nasim Alem, ${ }^{3}$ Himani Arora $\odot,{ }^{4}$ Sibylle Gemming, ${ }^{4}$ Artur Erbe, ${ }^{4}$ \\ Alex Zettl, ${ }^{5}$ Bernd Büchner, ${ }^{1}$ and Axel Lubk ${ }^{1, \dagger}$ \\ ${ }^{1}$ Institute for Solid State Research, IFW Dresden, Helmholtzstrasse 20, 01069 Dresden, Germany \\ ${ }^{2}$ Corrected Electron Optical Systems GmbH, Englerstrasse 28, 69126 Heidelberg, Germany \\ ${ }^{3}$ Department of Materials Science and Engineering, Pennsylvania State University, N-210 Millennium Science Complex, \\ University Park, Pennsylvania 16802, USA \\ ${ }^{4}$ Helmholtz-Zentrum Dresden-Rossendorf, 01328 Dresden, Germany \\ ${ }^{5}$ Department of Physics, University of California Berkeley, 366 LeConte Hall MC 7300, Berkeley, California 94720-7300, USA
}

(Received 1 July 2020; revised 6 October 2020; accepted 27 October 2020; published 11 December 2020)

\begin{abstract}
The reduced dimensionality in two-dimensional materials leads to a wealth of unusual properties, which are currently explored for both fundamental and applied sciences. In order to study the crystal structure, edge states, the formation of defects and grain boundaries, or the impact of adsorbates, high-resolution microscopy techniques are indispensable. Here we report on the development of an electron holography (EH) transmission electron microscopy (TEM) technique, which facilitates high spatial resolution by an automatic correction of geometric aberrations. Distinguished features of EH beyond conventional TEM imaging are gap-free spatial information signal transfer and higher dose efficiency for certain spatial frequency bands as well as direct access to the projected electrostatic potential of the two-dimensional material. We demonstrate these features with the example of $h$-BN, for which we measure the electrostatic potential as a function of layer number down to the monolayer limit and obtain evidence for a systematic increase of the potential at the zig-zag edges.
\end{abstract}

DOI: 10.1103/PhysRevResearch.2.043360

\section{INTRODUCTION}

The discovery of graphene and its intriguing properties more than ten years ago [1,2] has sparked large and ongoing research efforts into two-dimensional materials (2DMs). The synthesis of novel 2DMs, comprising, e.g., 2D topological insulators, 2D magnets, or organic systems like 2D polymers, with single to few layer thickness and high structural definition at the atomic/molecular level, is at the center of this field (e.g., [3-6]). They exhibit a large range of physical properties triggered by the reduced dimensionality in one direction such as quantum confinement effects or weak dielectric screening from the environment, yielding a significant enhancement of the Coulomb interaction [3,7]. Another interesting aspect is the formation of out-of-plane elastic modulations, which stabilize the 2DM structure and modify its mechanical properties [8-10]. The missing third dimension also enhances the proliferation and impact of defects, such as point and line defects, grains, or multilayer morphologies, which inevitably occur upon synthesis and often govern the functionality (e.g., reactivity and stability) of 2DMs in applications [11].

\footnotetext{
*Corresponding author: f.1.kern@ifw-dresden.de

${ }^{\dagger}$ Corresponding author: a.lubk@ifw-dresden.de
}

Published by the American Physical Society under the terms of the Creative Commons Attribution 4.0 International license. Further distribution of this work must maintain attribution to the author(s) and the published article's title, journal citation, and DOI.
Therefore the development of microscopic characterization methods, which allow one to analyze the structure and electronic properties of the 2DMs including the edges, defects, and grain boundaries, is at the center of the field. Transmission electron microscopy (TEM) has been a cornerstone technique (others are scanning tunneling microscopy and photoemission electron microscopy), offering high resolving power and spectroscopic information. A breakthrough for TEM could be achieved by employing chromatic aberration correction facilitating high-spatial resolution at low-acceleration voltages [12]. Two central challenges required special attention and have been addressed through various methodological developments of TEM techniques.

(A) 2D materials are typically more susceptible to various beam damage mechanisms than their three-dimensional counterparts [13-15]. That includes knock-on damage, radiolysis, and chemical etching. The knock-on damage may be reduced by lowering the acceleration voltage and hence the kinetic energy of the beam electron below the knock-on threshold of the pertinent chemical bonds in the 2DM (e.g., $90 \mathrm{keV}$ for the C-C bond in graphene [16] and $40 \mathrm{keV}$ for the B-N bond in monolayer $h$-BN [17]). Radiolysis and etching follow more complicated reaction mechanisms [18]. Their magnitude might be reduced by lowering the temperature and optimizing the acceleration voltage [16].

(B) Most 2D materials belong to the family of weak scatterers (another important member is biological matter), which implies that they only (weakly) shift the phase of the electron wave when traversing the sample, but do not modulate the amplitude. Consequently, they are referred to as weak 
phase objects (WPOs). This phase shift of the electron wave cannot be measured directly, due to the quantum mechanical phase problem. To solve this problem one can employ phase plates, which enable the imaging of the phase shift introduced by these materials as intensity contrast. The use of either physical [19-22] or electron optical phase plates [19,23-25], however, comes with some merits and disadvantages. The former degrade $[21,22,26]$ during use and create unwanted diffuse scattering and beam blocking [27], whereas the latter are typically constructed from materials that are prone to charging. Notable exceptions are laser [28] and drift tube [25] phase plates, which are very demanding constructionwise and implementationwise. By far the most straightforward method for transfer of phase contrast to intensity contrast, however, is an additional defocus with respect to the object exit plane, which has the negative side effect of introducing transfer gaps at low spatial frequencies or an oscillating contrast transfer for large spatial frequencies (see below). Introducing large defoci also results in a reduction of the resolution due to the partial transversal coherence of the electrons, which may be expressed by an exponential envelope function in reciprocal space.

In the following we address the phase problem in weak scatterers (for the example of 2DMs) by advancing off-axis electron holography, an interferometric technique allowing one to reconstruct the phase shift of the electron wave over the whole spatial frequency band, up to the information limit. These advantages have triggered a small number of previous studies on WPOs, notably for biological materials [29,30] and 2DMs [31-36]. However, a persisting problem remains in the defocus required for visualizing the sample during TEM operation and other residual aberrations such as astigmatism, which typically build up during acquisition [37]. Their correction, however, is mandatory for an analysis of the acquired phase in terms of physical quantities such as potentials, charge densities, and the atomic structure. In Winkler et al. [35,36] this problem has been addressed by a model-based fitting approach requiring a full model of the scattering potential and hence the 2DM under investigation. Boureau et al. [38] reconstructed the electrostatic potential and charge density of an $\mathrm{MoS}_{2}$ monolayer from a series of holograms in order to increase to effective exposure time. They corrected drifts of defocus, twofold astigmatism, sample, and biprism in between the different exposures using the averaging scheme proposed by Niermann and Lehmann [39]; however, they did not provide information on how to determine the absolute values of the aberrations.

Here we follow a different approach requiring no or only minimal a priori knowledge of the sample, that is, stripping the recorded data from any instrumental influences, notably aberrations and noise. The resulting data may then be used to extract certain specimen properties in a second step. This approach has the advantage of requiring no a priori knowledge about the specimen and a clear separation between instrumental influences and characteristics of the specimen. One key idea is to exploit very general discrete symmetries pertaining to the scattered electron wave function: First of all, the weak scattering property induces an odd symmetry in the object phases in Fourier space, which allows one to correct for symmetric aberrations. Second, a large class of
2DMs is centrosymmetric, introducing an even symmetry in the Fourier object phases, which allows one to correct for antisymmetric aberrations. This approach is based on the original work of $\mathrm{Fu}$ et al. [40], who demonstrated how to generically extract symmetric aberrations from holograms for the example of amorphous carbon foils. Here, we go one step further and autocorrect for the aberrations in the reconstructed wave of 2DMs, which greatly facilitates the analysis of the phase in terms of physical data, i.e., projected potentials. In this regard, we follow Gabor's original idea of holography as a means to aberration correction [41]. The corrected data are then subjected to a principle component analysis (PCA) denoising, which reveals the meaningful phase data at the atomic scale and enables extracting the underlying projected potential. Last but not least we compare those data to $a b$ initio density functional calculations to analyze the measured potentials in terms of charge (de)localizations.

The paper is organized as follows. We first recapitulate the imaging principles of weak scatterers (phase objects) and off-axis holography. From these, we derive how geometric aberrations can be determined and automatically corrected a posteriori from an acquired hologram without additional measurements. We elaborate on the noise characteristics of the thereby obtained aberration-corrected image phase and the spatial resolution of the determined aberrations (e.g., defocus due to out-of-plane modulations). We finally demonstrate the feasibility of the approach for the example of $h$-BN. Amongst others we reconstruct the number of layers, the mean inner potential (MIP) of individual layers, and the structure of the monolayer as well as the edges, and correlate this to material properties such as the charge delocalization or the stability and electronic properties of the edge states.

\section{IMAGING PRINCIPLES}

\section{A. Conventional imaging and off-axis holography of a weak phase object}

Sufficiently thin TEM specimens (with the critical thickness depending on the atomic scattering potential $V$ of the chemical constituents) behave as WPOs in TEM. In good approximation they just impose a small phase shift

$$
\varphi_{\mathrm{obj}}(\boldsymbol{r})=C_{\mathrm{E}} \int_{-t / 2}^{+t / 2} V(\boldsymbol{r}, z) d z
$$

on the beam electrons' wave function $\Psi_{\text {obj }}$ leaving the object of thickness $t$. Here $C_{\mathrm{E}}$ is an electron-energy-dependent interaction constant $(0.01 \mathrm{rad} / \mathrm{V} \mathrm{nm}$ at $80 \mathrm{keV}), z$ is the direction in which the electron beam transmits the sample, and $\boldsymbol{r}$ is the 2D position vector in the object plane. Since WPOs do not modulate the amplitude $A, \Psi_{\text {obj }}$ can be approximated by

$$
\Psi_{\mathrm{obj}}(\boldsymbol{r}) \stackrel{(\mathrm{WPO})}{\approx} A_{\mathrm{obj}}\left[1+i \varphi_{\mathrm{obj}}(\boldsymbol{r})\right] .
$$

The dominant geometric aberrations of the objective lens are described by a phase function $\chi(\boldsymbol{k})$ acting on the electron wave spectrum by a complex factor $e^{-i \chi(k)}$, the wave transfer function (WTF), in reciprocal space. Moreover, the combination of (transversal and longitudinal) partial coherence and geometric aberrations leads to an exponential damping of spatial frequencies in the wave function described by a 
(a)

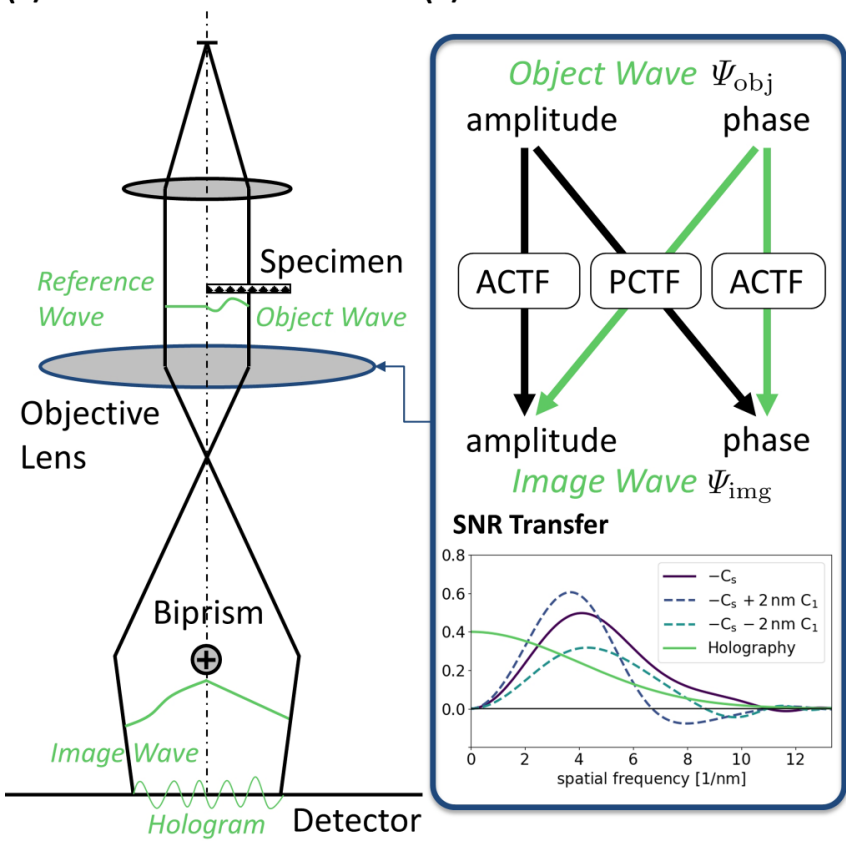

FIG. 1. (a) Off-axis electron holography (EH) setup. (b) Signal transfer for imaging a weak phase object (WPO) and signal-to-noise (SNR) transfer functions for HRTEM in negative $C_{\mathrm{s}}$ conditions $\left(-C_{\mathrm{s}}\right)$ and EH [see Eq. (9)]. In case of HRTEM, only the signal transfer from object phase to image amplitude described by the phase contrast transfer function (PCTF) contributes to the image. In case of EH, also the signal transfer from object phase to image phase described by the amplitude contrast transfer function (ACTF) contributes to the image. Additionally, the corresponding transferred bands for HRTEM in $-C_{\mathrm{s}}$ conditions with \pm 2 -nm defocus variation are also plotted. A defocus drift of about $2 \mathrm{~nm}$ is commonly observed after about $5 \mathrm{~min}$ in aberration-corrected TEM instruments [37].

real envelope function $E(\boldsymbol{k})$ in reciprocal space. The Fourier transform of the so-called image wave function taking into account these modulations by the imaging system [Fig. 1(a)] reads

$$
\begin{aligned}
\tilde{\Psi}_{\mathrm{img}}(\boldsymbol{k}) & =\tilde{\Psi}_{\mathrm{obj}}(\boldsymbol{k}) e^{-i \chi(\boldsymbol{k})} E(\boldsymbol{k}) \\
& \stackrel{(\mathrm{WPO})}{\approx} \mathrm{A}_{o b j}\left[\delta(\boldsymbol{k})+i e^{-i\left[\chi_{s}(\boldsymbol{k})+\chi_{a}(\boldsymbol{k})\right]} E(\boldsymbol{k}) \tilde{\varphi}_{\mathrm{obj}}(\boldsymbol{k})\right] .
\end{aligned}
$$

Here we separated the antisymmetric and symmetric aberrations, $\chi_{\mathrm{a}}(\boldsymbol{k})$ and $\chi_{\mathrm{s}}(\boldsymbol{k})$, respectively. The corresponding conventional linear image intensity (neglecting the term quadratic in $\varphi_{\mathrm{obj}}(\boldsymbol{r})$ and additional smearing due to the detector) reads

$$
\begin{aligned}
I(\boldsymbol{r}) & =\left|\Psi_{\mathrm{img}}(\boldsymbol{r})\right|^{2} \\
& \approx A^{2}+2 A^{2} \mathcal{F}^{-1}\{\underbrace{\sin \chi_{\mathrm{s}}(\boldsymbol{k}) E(\boldsymbol{k})}_{\text {PCTF }} e^{-i \chi_{\mathrm{a}}(\boldsymbol{k})}\} * \varphi_{\mathrm{obj}}(\boldsymbol{r}) .
\end{aligned}
$$

Here we observe that only the so-called phase contrast transfer function (PCTF) containing the symmetric aberrations produces visible contrast by convolution $(*)$ with the phase [Fig. 1(b)]. Several strategies have been developed to optimize the transfer over certain spatial frequency bands.
Most notably, in state-of-the-art instruments equipped with hardware-aberration correctors, the spherical aberration $\left(C_{\mathrm{s}}\right)$ and defocus can be traded to produce a positive contrast transfer for high-resolution TEM (HRTEM) over a band, ultimately limited by the incoherent chromatic envelope of the instrument (also referred to as negative $C_{\mathrm{s}}$ imaging conditions [42]). The latter limitation could be largely eliminated by employing chromatic aberration correctors [43], ultimately leading to an image spread limited resolution (due to Johnson noise [44])

$$
E(\boldsymbol{k})=\exp \left(-2 \pi^{2} \sigma_{\mathrm{i}}^{2} k^{2}\right)
$$

in such instruments [Fig. 1(b)]. Upon inspection of the PCTF it becomes immediately clear that, if negative $C_{\mathrm{s}}$ conditions are perfectly adjusted, it acts as a bandpass in HRTEM conditions, mainly suppressing small spatial frequencies $\left[-C_{\mathrm{S}}\right.$ in Fig. 1(b)]. This property complicates for instance the analysis of the 2DM's morphology such as determining the layer number representing large scale spatial structures (see, e.g., [45], for example on $h$-BN).

While the transfer of large spatial frequencies may be only increased through further improved electron optics and/or reconstructing multiple images with varying imaging conditions (e.g., focal series [46], beam tilt series [47], or ptychography [48]), both large and small spatial frequencies can be transferred simultaneously by employing off-axis holography. Here, the object is inserted halfway into the beam path, which automatically restricts the field of view to edges of the 2D material, whereas the other half space is occupied by the undisturbed reference wave. Both parts are brought to superposition by employing an electrostatic Möllenstedt biprism, forming a hologram in the image plane [Fig. 1(a)]. From the latter a complex wave function

$$
\tilde{\Psi}_{\mathrm{hol}}(\boldsymbol{r})=\mathcal{F}^{-1}\left\{\mu(\boldsymbol{k}) \tilde{\Psi}_{\mathrm{img}}(\boldsymbol{k})\right\}
$$

is reconstructed [49] (see Appendix B for details). Here, the contrast factor $\mu=\mu_{\mathrm{c}}$ MTF takes into account the detector modulation transfer function (MTF) of the detector and the illumination degree of coherence, inelastic scattering, and instrumental instabilities (wrapped up in $\mu_{c}$ ). Since the whole wave function is reconstructed only the incoherent envelopes $E(\boldsymbol{k})$ limit the transfer; in particular there is no damping of small spatial frequencies by the PCTF. For simplicity we will approximate the contrast factor $\mu(\boldsymbol{k})$ with that of the hologram carrier frequency $\mu\left(\boldsymbol{k}_{\mathrm{c}}\right)$ in the following.

In addition, the linear reconstruction principle allows one to compute the noise transfer and hence the error (in terms of variance) pertaining to the reconstructed phase from the noise transfer function of the detector [50,51]. If we use this result and compare conventional HRTEM of WPOs with off-axis holography in terms of signal-to-noise ratio (SNR) of the phase contrast for a particular spatial frequency (see Appendix $\mathrm{C}$ for a detailed derivation), we obtain

$$
\frac{\mathrm{SNR}_{\mathrm{hol}}}{\mathrm{SNR}_{\mathrm{conv}}}=\frac{\mu_{\mathrm{c}}\left(\boldsymbol{k}_{\mathrm{c}}\right) \sqrt{\mathrm{DQE}(0)}}{\sin \chi_{\mathrm{s}} \sqrt{\overline{\mathrm{DQE}\left(\boldsymbol{k}_{\mathrm{c}}\right)}}} .
$$

Here, DQE denotes a 2D generalization of the detection quantum efficiency as detailed in Appendix C. If this ratio becomes 
larger than 1, i.e.,

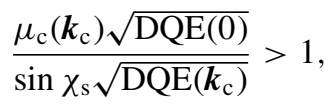

off-axis holography is more dose-efficient than conventional phase contrast in terms of retrievable information per dose. Noting that a realistic value for the fringe contrast in highresolution holograms recorded at modern TEMs equipped with state-of-the-art detectors and field-emission guns can reach several tens of percents (in this paper $30 \%$, see below), this condition is met in a broad range of low to medium spatial frequencies [see Fig. 1(b)] but not for high spatial frequencies. Note, however, that the slight inferiority of off-axis holography at high spatial frequencies compared to HRTEM may be overcome by the use of novel direct counting detectors with largely reduced detector DQEs [52,53].

\section{B. A posteriori correction of residual aberrations}

Following $\mathrm{Fu}$ et al. [40] the symmetric aberrations can be readily extracted from the phases $\tilde{\varphi}_{\text {img }}$ of the image wave function in reciprocal space [Eq. (3)]:

$$
\chi_{s}(\boldsymbol{k})=-\frac{1}{2}\left[\tilde{\varphi}_{\mathrm{img}}(\boldsymbol{k})+\tilde{\varphi}_{\mathrm{img}}(-\boldsymbol{k})\right]+\frac{\pi}{2}+\pi n(\boldsymbol{k}),
$$

which follows from the antisymmetry of Fourier phases of the original WPO (see Appendix A for a detailed derivation). Here the appearance of the integer $(n \in \mathbb{N}) \pi$ ambiguity stems from the $2 \pi n$ ambiguity of the original wrapped phases. The above relation is remarkable as it allows one to compute (and therefore correct) the symmetric part of the phase plate $\chi(\boldsymbol{k})$ (aberrations) without any a priori knowledge about the object or the incoherent envelopes including the detector MTF. The only condition for a successful practical application is that the object spectra SNR must be large enough to suppress error propagation of inevitable reconstructed noise and other artifacts (e.g., Fresnel fringes).

A similar expression cannot be derived for the antisymmetric aberrations, because they do not produce an amplitude variation from the WPO [see Eq. (5)]. Similar to the wellknown Zemlin tableau method [54], they can be determined from a tilt series (where lower-order symmetric aberrations are induced by higher-order antisymmetric ones), or additional symmetry criteria. In particular, for the large class of centrosymmetric 2DMs we have $\tilde{\varphi}_{\mathrm{a}}=0, \pi$ and hence

$$
\chi_{\mathrm{a}}(\boldsymbol{k})=-\frac{1}{2}\left[\tilde{\varphi}_{\mathrm{img}}(\boldsymbol{k})-\tilde{\varphi}_{\mathrm{img}}(-\boldsymbol{k})\right]+\pi n(\boldsymbol{k}) .
$$

Here, $(n \in \mathbb{N}) \times \pi$ ambiguity stems from the $\pi$ phases of the object.

To finally correct for the aberrations from the holographically reconstructed wave functions, we multiply its Fourier transform with the complex conjugate of the WTF, i.e.,

$$
\tilde{\Psi}_{\mathrm{obj}}(\boldsymbol{k}) E(\boldsymbol{k})=\tilde{\Psi}_{\mathrm{img}}(\boldsymbol{k}) e^{i \chi(\boldsymbol{k})} .
$$

Note, however, that this involves a phase unwrapping procedure removing the $\pi$ ambiguity in the phase plate, which can be challenging in practice, depending on the spectrum of the object wave. This currently limits the scope of the autocorrection scheme to precorrected imaging conditions (e.g., using hardware corrected TEMs), where only small residual
TABLE I. Holographic imaging conditions at the TEAM I microscope adjusted for electron wave reconstruction of two-dimensional materials.

Acceleration voltage $U_{\mathrm{a}}$

$80 \mathrm{kV}$

$C_{\mathrm{c}}$ and $C_{\mathrm{s}}\left(C_{3}\right)$

$<10 \mu \mathrm{m}$

Image spread $\sigma_{\mathrm{i}}$

Information limit

$40 \mathrm{pm}$

Diffraction lens excitation

$0.13 \mathrm{~nm}$

$65 \%$

Pixel size of hologram

$0.054 \mathrm{~nm}$

Mean counts per hologram pixel $I_{\text {hol }}$

Biprism voltage $U_{\mathrm{bi}}$

Fringe visibility $\mu\left(\boldsymbol{k}_{\mathrm{c}}\right)[50]$

$\operatorname{DQE}\left(\boldsymbol{k}_{\mathrm{c}}\right)$ [50]

$\approx 10000$

$160 \mathrm{~V}$

0.3

0.5

aberrations and sufficiently small defoci are present, keeping the phase range within $\pi$ over a large band.

The above considerations are strictly correct for the WPO only. In praxis, this condition may be violated to some extent, e.g., when employing low acceleration voltages (resulting in higher phase shifts) to reduce knock-on damage in a certain class of 2DMs. Note, however, that the "constant-amplitude" criterion also applies to pure phase objects and may be even slightly generalized to weak amplitude objects by minimizing a penalty term for the amplitude variations, e.g.,

$$
\chi(\boldsymbol{k})=\arg \min \left\|\nabla\left|\tilde{\Psi}_{\mathrm{img}}(\boldsymbol{k}) e^{i \chi(\boldsymbol{k})}\right|\right\| .
$$

Lehmann [55] and Ishizuka et al. [56] reported different approaches to this aberration assessment via direct amplitude variation minimization for WPOs. It is currently an open question, whether and under which conditions this generalization yields unique solutions [57].

\section{EXPERIMENTAL}

To validate the autocorrection method we apply the above machinery to a single to few atomic layer van der Waals 2DM, namely, hexagonal boron nitride $(h-\mathrm{BN}) . h$-BN has a crystal structure very similar to that of graphene [see Fig. 2(i)], but possesses completely different electronic properties (notably a large band gap and no Dirac points) [58,59]. The sample was prepared using an exfoliation and reactive ion etching process similarly to Ref. [45], with the only difference that, instead of plasma-cleaning the sample, the latter was cleaned and thinned inside the TEM instrument by an electron-beam shower of about $20 \mathrm{nA}$. The electron holograms have been recorded at a chromatic aberration $\left(C_{\mathrm{c}}\right)$ corrected TEM instrument, the TEAM I at the Molecular Foundry at the National Berkeley Laboratory, using the imaging conditions listed in Table I. The $C_{\mathrm{c}}$ correction, notably, allowed us to resolve the $\{2110\}$ family of spatial frequencies not visible in a conventional $C_{\mathrm{s}}\left(C_{3}\right)$ corrected electron microscope. A 20-min-long time series of holograms was acquired using a $2 \times 2-\mathrm{k} C C D$ camera (Model 894 US1000, Gatan, Inc.), each with 8-s exposure time owing to the great instrumental stability of the microscope in order to enhance the SNR. For further analysis we chose a single hologram from the time series, out of those that do not exhibit notable sample drift during the exposure. We had to slightly defocus the $h$-BN sample plane to have 

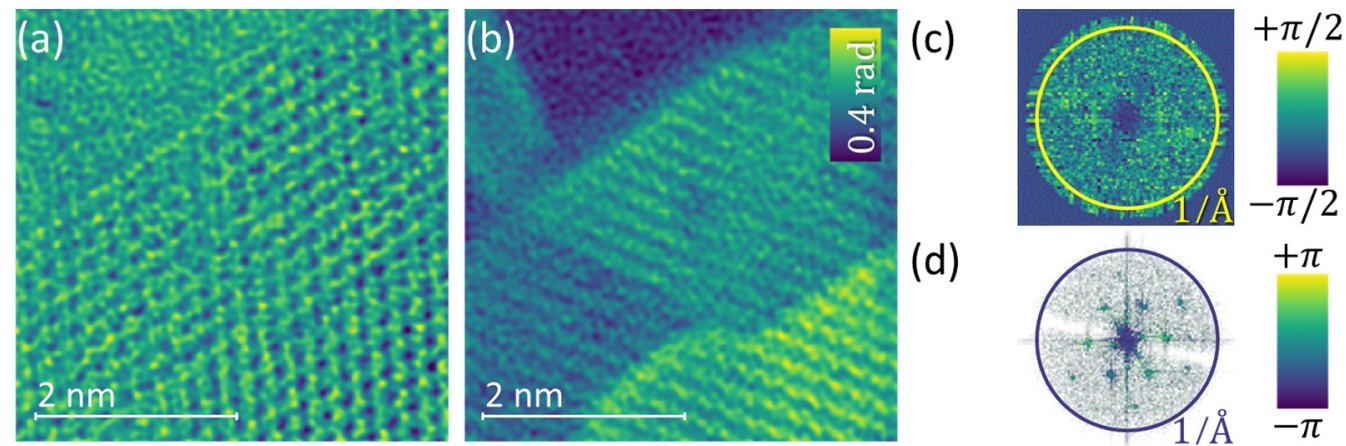

(d)
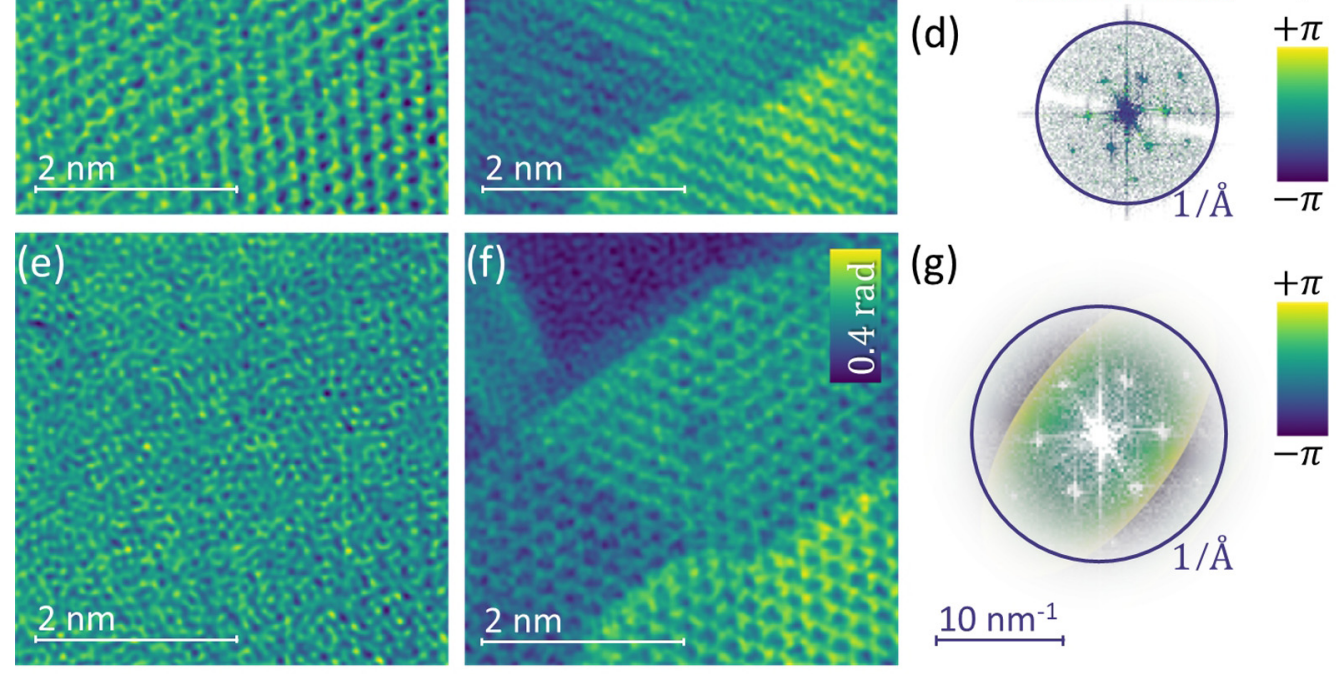

(g)

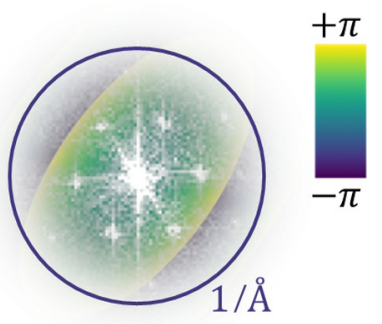

$10 \mathrm{~nm}^{-1}$
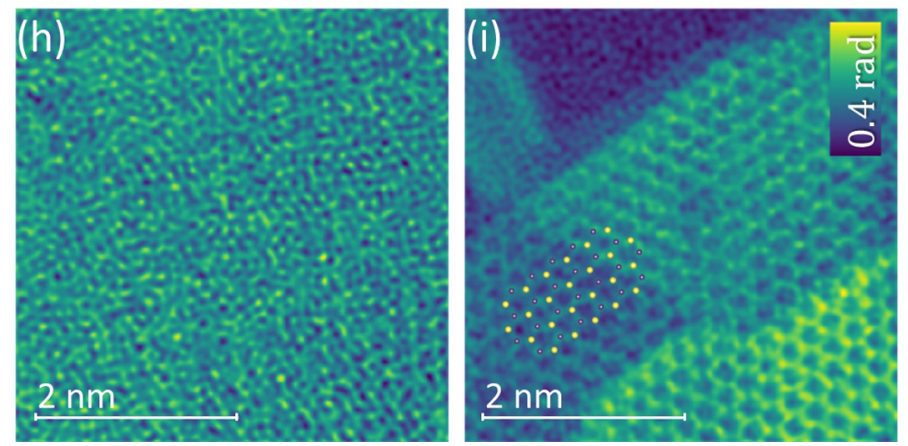

(j)

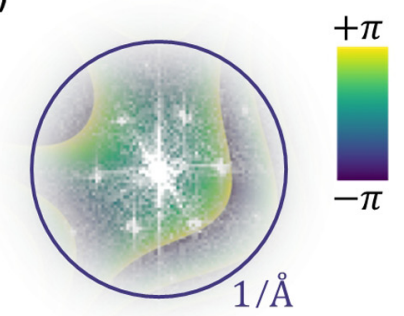

$10 \mathrm{~nm}^{-1}$

FIG. 2. Aberration correction of the $h$-BN image wave reconstructed by off-axis electron holography. (a), (b) As-reconstructed wave image in amplitude and phase. The symmetric aberration phase plate (c) as determined numerically from Eq. (11) displays all spatial frequencies, including those where noise is dominating because no object information is present. (d) The color-coded representation of panel (c) is overlayed with the Fourier spectrum of the image wave (a), (b) to highlight the meaningful part of the numerical phase plate. (e), (f) Amplitude and phase corrected for symmetric aberrations using the continuous phase plate (g) fitted from panel (c). (h), (i) Amplitude and phase corrected also for antisymmetric aberrations (coma and threefold astigmatism) obtained from Eq. (12) using the phase plate (j). The crystal structure of a $h$-BN monolayer is indicated in panel (i).

sufficient contrast for selecting the desired object position into the field of view. We note that the defocus, as well as the twofold astigmatism, were considerably drifting and that the electron induced charging of the sample is changing at a modest level over the time frame of the series. Significant, presumably knock-on induced beam damage can be observed over the $20 \mathrm{~min}$, especially at the boundary to vacuum. The recorded holograms were then processed off-line through a removal of dead and hot pixels by an iterative local threshold algorithm, as well as a masking out of Fresnel fringes [60]. In addition, a deconvolution of the CCD camera's MTF and a modest Wiener filtering [57] in Fourier space were employed to increase the SNR of the holograms [57]. Within the holographic Fourier reconstruction method [49], one sideband was masked with a circular tenth-order Butterworth filter with a radius of $8.5 \mathrm{~nm}^{-1}$. The phase of the reconstructed wave was subtracted by the phase reconstructed from an additionally recorded and equally processed object-free empty hologram, to correct for distortions induced by the fiber optics that couple the scintillator to the CCD camera. The as-reconstructed amplitude and phase of a small region of overlapping $h$-BN sheets are depicted in Figs. 2(a) and 2(b). Since the sample is defocused, one observes an amplitude contrast described by the PCTF (see also Fig. 6 for the HRTEM image, i.e., reconstructed centerband). Moreover, a rather large twofold astigmatism and other residual aberrations seem to be present, rendering a quantitative analysis almost impossible. We now apply the autocorrection procedure outlined in Sec. II B. Figures 2(c)-2(j) show the results of the two autocorrection steps; the correction of symmetric aberrations from the WPO property [Figs. 2(e) $-2(\mathrm{~g})]$ and the final correction including also antisymmetric aberrations after exploiting the centrosymmetry of the $h$-BN lattice [Figs. 2(h)-2(j)]. Clearly, the amplitude [Fig. 2(e)] is almost constant after removal of symmetric aberrations [Figs. 2(a) and 2(e) are displayed within the same color levels], proving the experimental feasibility 
of the first autocorrection step. The numerical phase plate [Fig. 2(c)] computed using Eq. (11) provides a good SNR only where the reciprocal space is filled with specimen information [i.e., $h$-BN systematic reflections, Fig. 2(d)]. These are, however, sufficient to determine the geometrical aberration coefficients (within 95\% confidence intervals) of first-order aberrations, namely, defocus, $C_{1}=4.0 \pm 0.8 \mathrm{~nm}$, and twofold astigmatism, $\left\{A_{1}=2.5 \pm 1.1 \mathrm{~nm}, \alpha_{1}=54^{\circ} \pm 25^{\circ}\right\}$, by fitting a smooth polynomial

$$
\chi_{\mathrm{s}}=\frac{2 \pi}{k_{0}} k^{2}\left[C_{1}+A_{1} \cos \left(2 \alpha-\alpha_{1}\right)\right]
$$

[Fig. 2(g)] with the help of a Levenberg-Marquardt algorithm (third-order symmetric aberrations are small and could safely be neglected). We note that the correction with the numerically obtained phase plate [Fig. 2(c)] yields almost identical results as the correction with the corresponding fitted phase plate [Fig. 2(g)].

As stated above, the determination of the numerical antisymmetric phase plate is merely possible for centrosymmetric samples. Ignoring the small difference in atomic species, $h$ BN fulfills this symmetry condition for two different points, the center of the BN hexagons and the midpoints between the binding atoms. In order to find the most centrosymmetric region of interest, the symmetric aberrations corrected phase was split up into subimages, containing about 25 unit cells. Subsequently, for all of them, a numerical measure for the deviation from centrosymmetry was calculated. The most centrosymmetric subimage was finally used to determine the antisymmetric phase plate from Eq. (12), from which the corresponding phase plate [Fig. 2(j)]

$$
\chi_{a}=2 \pi \frac{k^{3}}{k_{0}^{2}}\left[\frac{1}{3} A_{2} \cos \left(3 \alpha-\alpha_{A_{2}}\right) \frac{1}{1}+B_{2} \cos \left(\alpha+\alpha_{B_{2}}\right)\right]
$$

with a threefold astigmatism of $\left\{A_{2}=158 \pm 261 \mathrm{~nm}\right.$, $\left.\alpha_{2}=-90^{\circ} \pm 16^{\circ}\right\}$ and an axial coma of $\left\{B_{2}=67 \pm 49 \mathrm{~nm}\right.$, $\left.\alpha_{\mathrm{B}_{2}}=-20^{\circ} \pm 54^{\circ}\right\}$ was fitted. Note that the $95 \%$ confidence intervals computed from the fit residual are rather large in this case, which is due to the relatively small size of the symmetric patch used for the fitting procedure.

In order to prepare the autocorrected high-resolution phase data for interpretation we apply PCA denoising in a final step (Fig. 3). Again, no a priori information about the material is required for this procedure. We rather exploit the regular geometric structure consisting of repeating honeycombs to create statistical data, which can be treated by PCA (i.e., modelfree) denoising [61]. Our procedure consists of cutting out patches slightly larger than one honeycomb, centering them, and subjecting the stack of patches to a PCA (see Appendix F for details). Inspecting the scree plot [Fig. 3(e)] we identified 11 non-noise components and truncated the data accordingly. The thereby denoised image is shown in Fig. 3(b) together with the original data [Fig. 3(a)]. The deviations to the original data [Fig. 3(d)] exhibit a noise-like Gaussian distribution [Fig. 3(c)] with the same standard deviation as the original phase noise in vacuum. Moreover, it shows no particular structure except at the edges, where fluctuations due to radiation damage occur during acquisition. We finally note that the phase noise $\sigma_{\varphi}$ of the original data [Fig. 3(a)] is consistent with that observed in the conventional HRTEM image data $\sigma_{\mathrm{I}}$ (obtained from the noninterference terms of the recorded hologram) after rescaling $\sigma_{\varphi}^{2} \approx \mathrm{DQE}(0) \mathrm{DQE}\left(\boldsymbol{k}_{\mathrm{c}}\right) / \sigma_{\mathrm{I}}^{2} \mu_{\mathrm{c}}\left(\boldsymbol{k}_{\mathrm{c}}\right)^{2}$, experimentally validating the noise considerations leading to Eq. (9). Here, the phase noise is smaller than the noise in the "raw" phase image because of the slight Wiener filtering mentioned above.

\section{RESULTS AND DISCUSSION}

After successful application of the autocorrection and denoising procedure we can now analyze the phase maps in detail. The high-resolution data (Fig. 5) clearly reveal regions of well-ordered honeycomb lattice with a multilayer morphology including various defects and edge structures. There are also regions, notably the bridge and some of the edges, where no or only a smeared-out honeycomb lattice is visible (facets are discernible though). These coincide with strongly oscillating parts of the sample, which were vanishing during the time series due to the electron irradiation. We therefore ascribe the loss of the high-spatial frequency data in this region to local vibrations rather than some sort of amorphization. Similar observations and quantification of the lattice distortion at the edges and vacancies have also been observed by quantitative phase contrast imaging as well as STEM imaging $[62,63]$.

Indeed, the quantitative phase data allow for a direct comparison with the projected potential of the $h$-BN lattice. We first focus on the low-frequency information (which might have been obtained also with medium-resolution holography modes and without the autocorrection of aberrations). To that end the high-resolution data are convoluted with a round top-hat function of the radius of a lattice constant $(0.25 \mathrm{~nm})$ and divided by $C_{\mathrm{E}}$ [see Eq. (1)] yielding the averaged projected Coulomb potential corresponding to the zeroth Fourier component of the potential in a periodic lattice. It depends sensitively on the charge (de)localization due to chemical bonding [64] (see also Appendix D) and is proportional to the diamagnetic susceptibility according to the Langevin theory [65] amongst others. In our case the average potential data reveal [Fig. 4(a)] the presence of different sample thicknesses, ranging from one to five atomic layers, visible as areas of constant projected potential, interrupted by a small number of defects. In the histogram Fig. 4(b) these regions can be associated to clear distinct peaks. Moreover, the differences of the peak positions determined by Gaussians fits yield the average potentials of each layer [Fig. 4(c)], that show a small decrease towards higher layer numbers. Noting that the average potential corresponds approximately to the sum of the second spatial moment (i.e., spatial extension, see Appendix D for a derivation) of the charge distribution of the contributing atoms, i.e., $\bar{V} \sim \sum\left\langle r^{2}\right\rangle_{\text {at }}$, this decrease in average potential could reflect a growing localization of the out-of-plane orbitals ( $3 p_{z}$ orbitals) in between $h$-BN layers as compared to the free surfaces. Indeed, the monoand bilayer average projected potential of about $4.5 \mathrm{~V} \mathrm{~nm}$ rather fit with independent atom potentials computed from Hartree-Fock [66] (4.4 V nm). The latter tend to be too delocalized compared to those computed from a full density functional theory (DFT) calculation (using FPLO-18 [67], see 

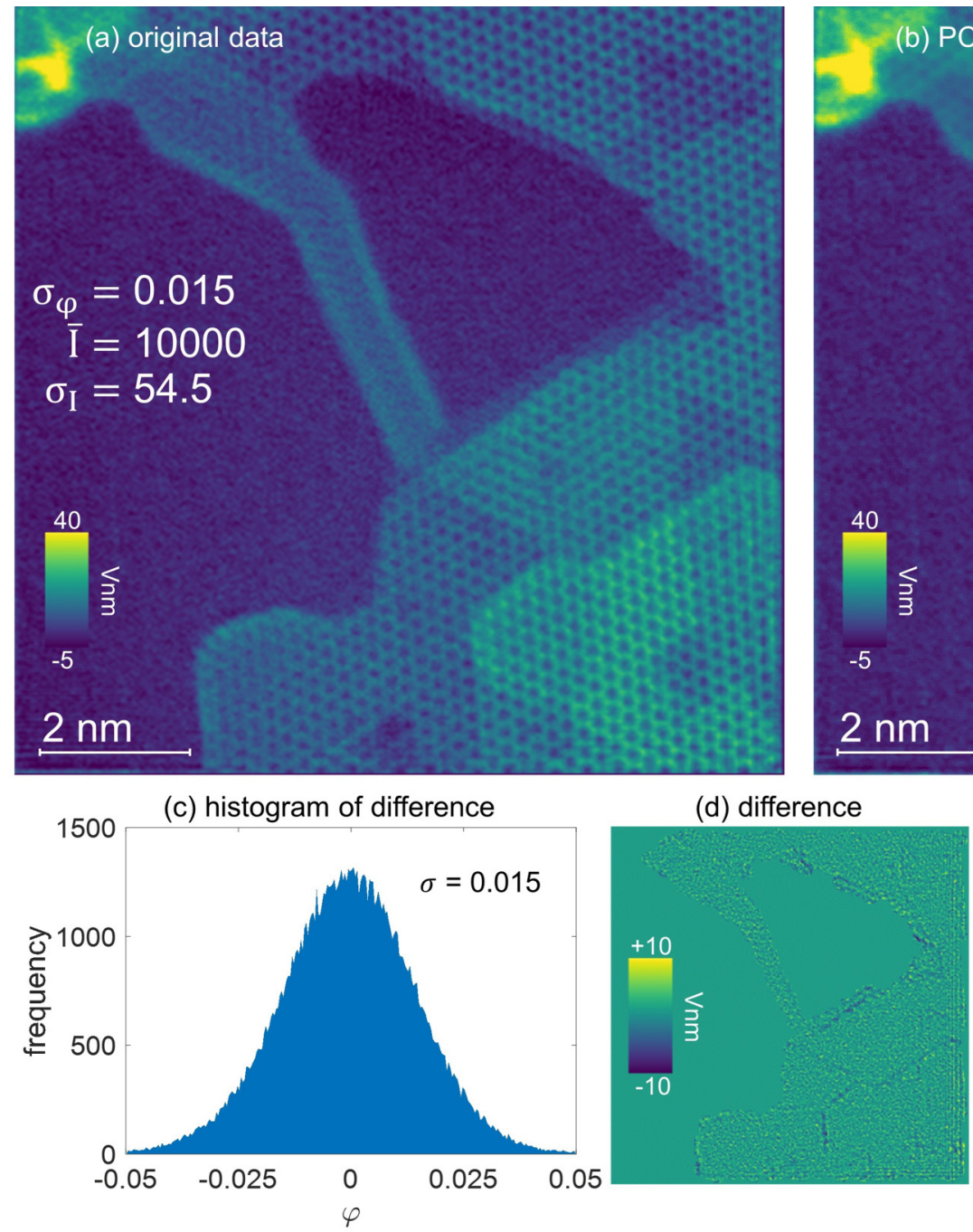

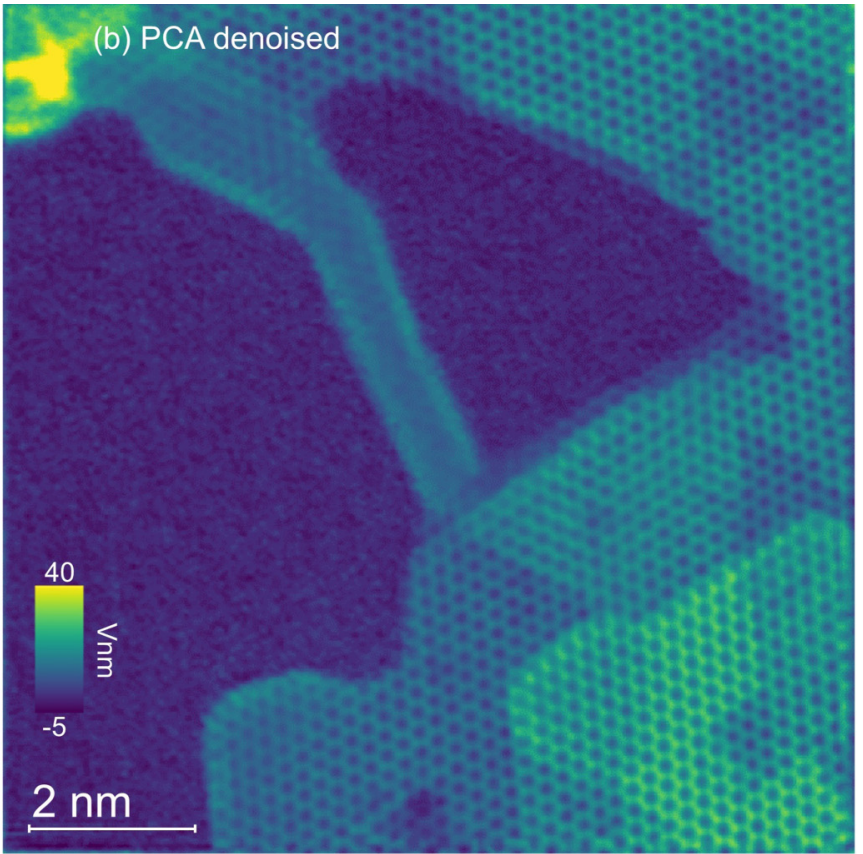

(e) scree plot

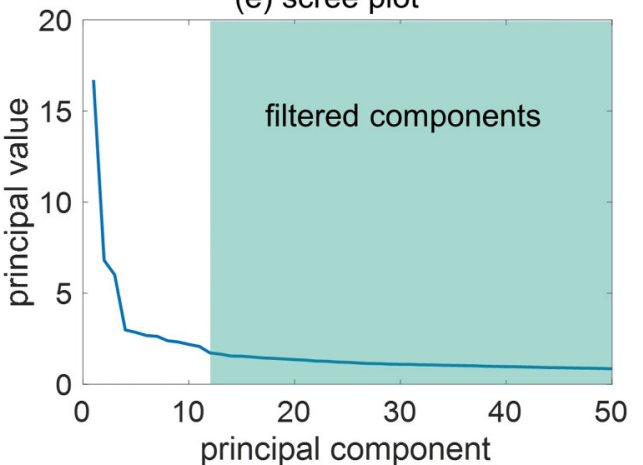

FIG. 3. Denoising of the aberration-corrected $h$-BN phase image reconstructed by off-axis electron holography. (a) Original autocorrected data and corresponding standard deviations of phase $\sigma_{\varphi}$ and intensity $\sigma_{\mathrm{I}}$ in vacuum. (b) PCA-denoised data after truncation to the first 11 principal components. The selection criterion is the last kink in the scree plot (e). Both the difference (d) and the histogram (c) reveal no noticeable deviation from Gaussian noise between the original and denoised image except at some structurally fluctuating edges.

Appendix E) including chemical bonds and correlation that agrees with a value of $3.4 \mathrm{~V} \mathrm{~nm}$ better to three and more layers [Fig. 4(c)]. Note furthermore that the structurally and atomweight-wise closely related graphene has a projected potential of $4.5 \mathrm{~V} \mathrm{~nm}$, which also reflects the stronger delocalization of the shell electrons in conducting graphene. Another possible explanation of the large potential values could be the positive charging of the (insulating) $h$-BN in the beam (ejection of secondary electrons).

A second noticeable feature is the potential increase visible at the edges and steps of the sample (most prominent in the double-layer bridge region). Different physical effects may be attributed to this potential elevation.

(1) Formation of (covalent) interlayer bonds at the zig-zag edges of bilayer $h$-BN, as proposed by Alem et al. [63], occurs through the following mechanisms: a local compression of the projected atomic positions or the tilting of the covalent B-N bonds out of plane, both of which yield a raised projected potential. These interlayer bonds could also explain the enhanced stability of even numbered layers under electron irradiation and thus their dominant appearance in the data set.

(2) Delocalization of in-plane $s, p_{\mathrm{x}}$, and $p_{\mathrm{y}}$ orbitals into vacuum could potentially lead to the observed increase of the average potential. Indeed, DFT calculations reported in literature predict the emergence of metallic edge states at the zig-zag edges $[68,69]$.

(3) Systematic adhesion of residual gas atoms occurs with different atomic potentials at the edges, such as oxygen [70].

Further studies are necessary to clarify and disentangle those effects quantitatively.

We now turn to the analysis of the autocorrected and denoised high-resolution data shown in Fig. 5(a). The denoised high-resolution data of the monolayer allow one to distinguish between the $\mathrm{B}$ and $\mathrm{N}$ sites in the monolayer [Fig. 5(d)]. Comparing the holographically measured potentials with the $a b$ initio potentials, which have been smeared out by multiplying the envelope function pertaining to the TEAM I instrument at $80 \mathrm{kV}$ [see Fig. 1(b)], we observe good agreement with an additional smearing of the ex- 

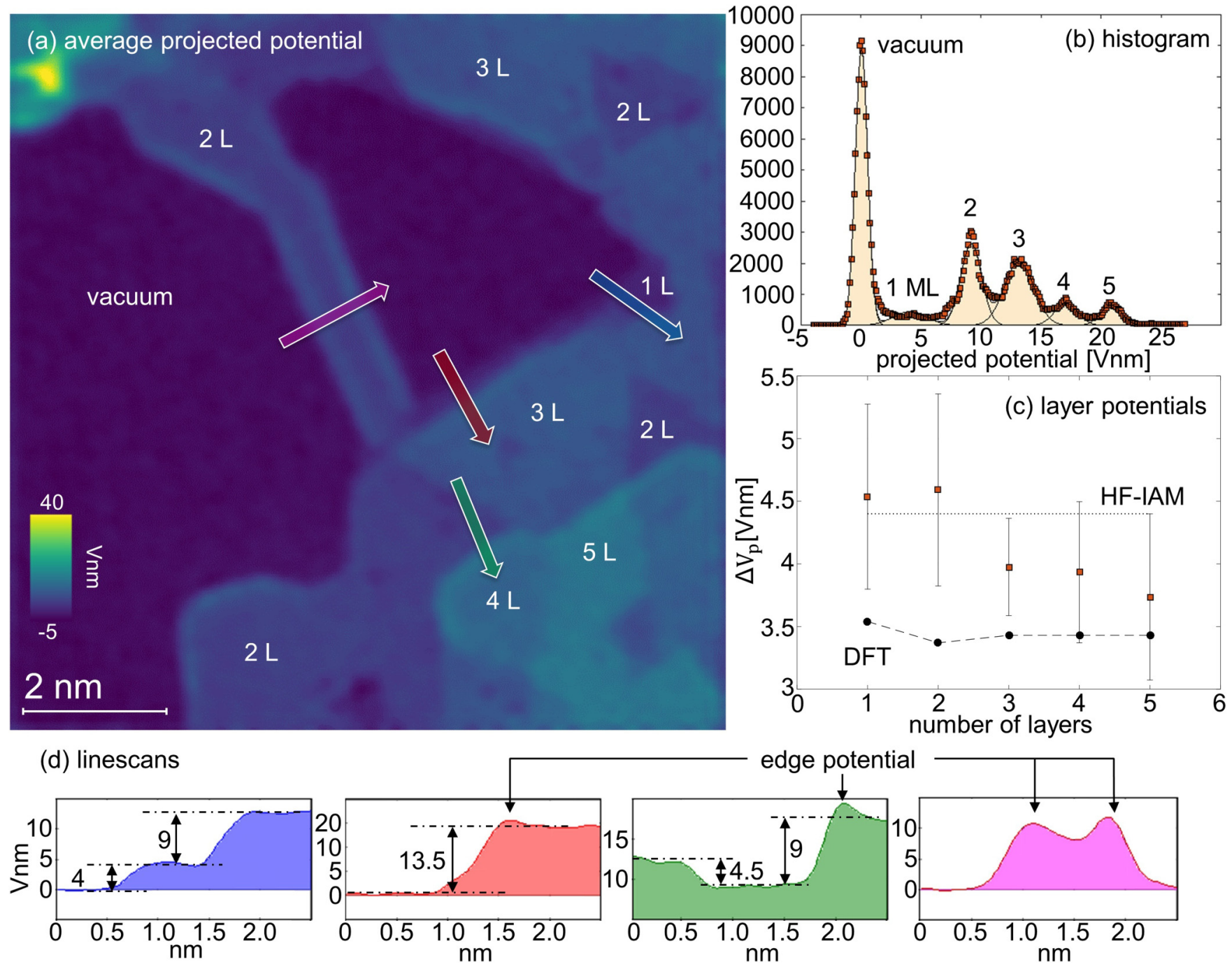

FIG. 4. Average potential analysis of $h$-BN. The overview image in panel (a) contains several flakes of different layer numbers, shaped by electron-beam irradiation. The potential data histogram in panel (b) reveals the presence of several monolayers, separated by well-defined potential offsets $\Delta V$ (c). The linescans in panel (d) provide local measurements for the latter and reveal the presence of potential bumps at edges and steps.

perimental data along the bonding directions [Fig. 5(f)]. Whether this is due to thermal vibrations (not included in our analysis) or other damping factors remains an open question at this stage. Turning to the edge structures, we observe that the zig-zag boundary is the prevalent configuration [Figs. 5(b) and 5(c)], which coincides with the $a b$ initio predictions [71]. We further note occasional distortions of the edge lattice, which may be attributed to some out-of-plane bending or ongoing beam damage (see $[62,63]$ ). Moreover, almost all edges reveal an increase in projected potentials, which has been already discussed above. That notably also includes steps [e.g., the step from two layer $(2 \mathrm{~L})$ to four layer $(4 \mathrm{~L})$ in Fig. 5(c)]. As noted previously we attribute these localized edge potentials to an increased electron delocalization, most probably due to a reconstruction of the edge structure along the $z$ direction including the formation of interlayer covalent bonds [63]. A detailed comparison to the emergence of particular edge states and the electronic configuration of defects [e.g., BN void depicted in Fig. 5(e)] is, however, beyond the scope of this paper and will be conducted elsewhere.

Summing up, we showed how autocorrected off-axis holography may be used as a high-resolution and doseefficient probe for 2DMs. In this regard we performed a parameter-free correction for antisymmetric aberrations based on the aforementioned symmetry principles. Notably the presented aberration autocorrection scheme and the favorable noise transfer properties of $\mathrm{EH}$ facilitate the reconstruction of quantitative potential data over a large spatial frequency band extending from zero to atomic resolution. The autocorrection scheme enables a removal of residual aberrations and defocus without the need to separately measure or fine tune them, rendering it a suitable method for in situ studies, where long time series need to be recorded. The high-structural order of 2DMs furthermore facilitated an efficient suppression of noise by adopting a PCA noise removal algorithm. Using these capabilities, we reveal several properties of $h$-BN. Notably, the electronic orbitals in $h$-BN are significantly more localized 


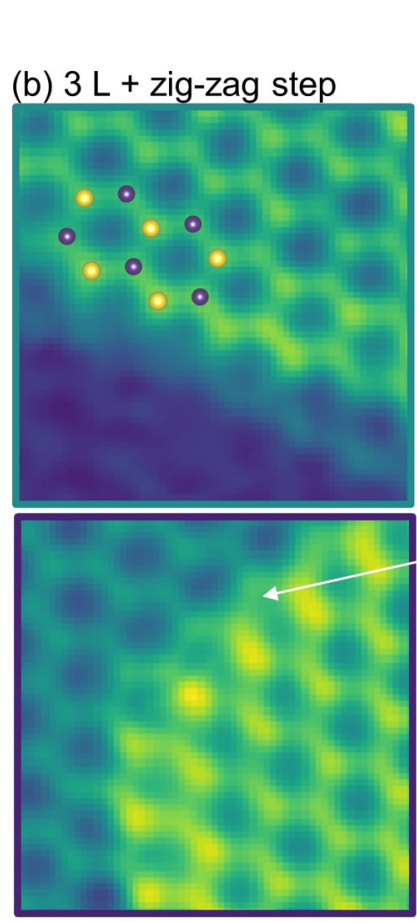

(c) $4 \mathrm{~L}+$ zig-zag step
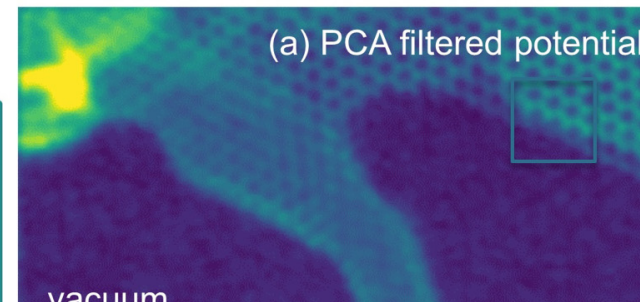

vacuum
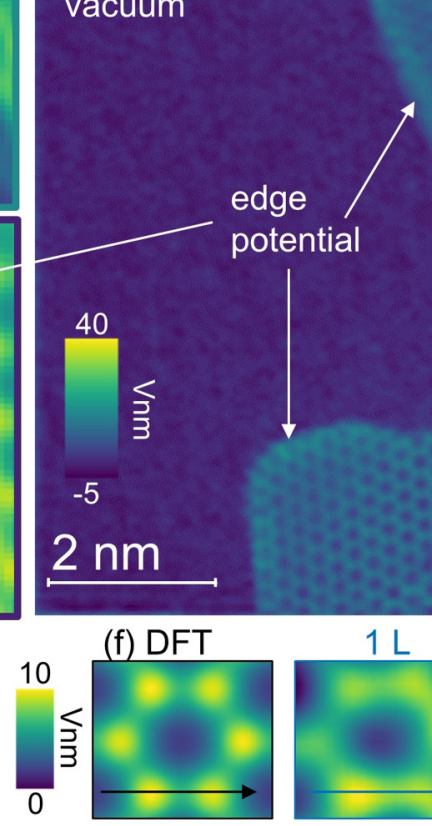

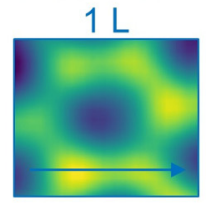

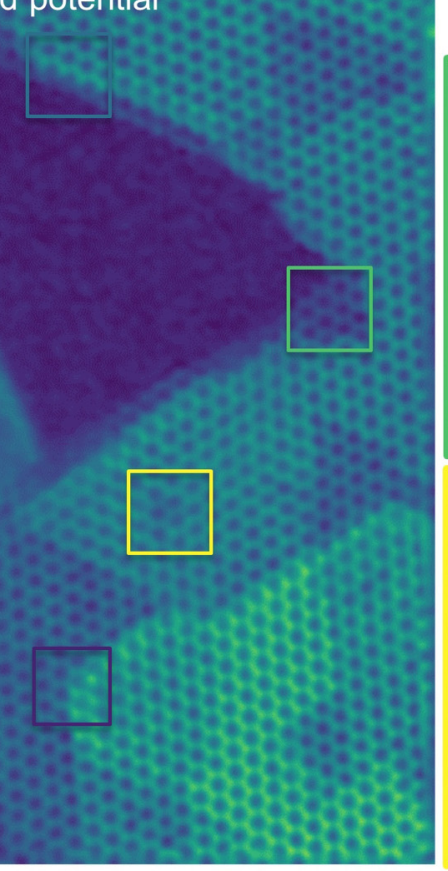

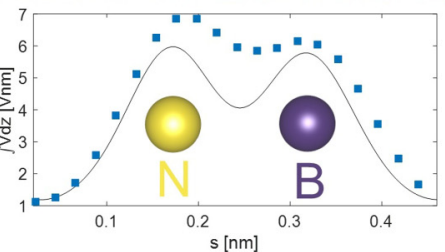

(d) $1 \mathrm{~L}$

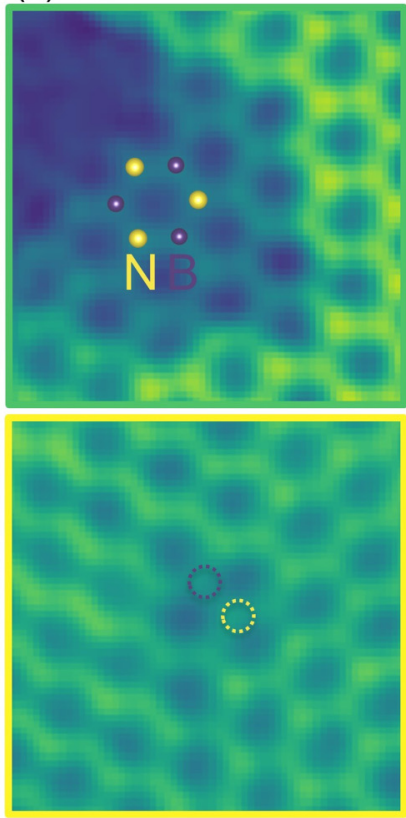

(e) $2 \mathrm{~L}+\mathrm{BN}$ void

FIG. 5. (a) High-resolution potential analysis. Insets: Zig-zag steps comprising two atomic layers (b), (c), a monolayer region (d), and a BN void defect (e). The monolayer potential is depicted together with the DFT result in panel (f).

as in the structurally similar graphene, resulting in a comparatively low mean projected potential of $3.6 \mathrm{~V} \mathrm{~nm}$. We could confirm that edges favor the zig-zag configuration and found a peculiar localized increase of the potential at the edges. The latter is attributed to the delocalization of electron edge states.

\section{ACKNOWLEDGMENTS}

We are grateful to Tore Niermann, who supported us at recording additional holograms of another $h$-BN sample. We acknowledge funding from the European Research Council under the Horizon 2020 research and innovation program of the European Union (Grant No. 715620) and the Deutsche Forschungsgemeinschaft (Project No. 417590517, Grant No. SFB 1415). This work is also supported by the Office of Science, Office of Basic Energy Sciences of the U.S. Department of Energy under Contract No. DE-AC02-05CH11231 and by the Initiative and Networking Fund of the Helmholtz Association of German Research Centers through the International Helmholtz Research School for Nanoelectronic Networks, IHRS NANONET (Grant No. VH-KO-606).

\section{APPENDIX A: WEAK PHASE OBJECT PHASE PLATES}

This Appendix contains derivations for the expressions (11) and (12) relating holographic data and aberrations. We start with rewriting the expression for the suitably normalized Fourier-transformed image wave (3) using $\tilde{\varphi}=A_{\tilde{\varphi}} e^{i P_{\tilde{\varphi}}}$ :

$$
\begin{aligned}
\frac{\tilde{\Psi}_{\mathrm{img}}-2 \pi \delta(\boldsymbol{k})}{A} & =\tilde{A}_{\mathrm{img}} e^{i \tilde{\phi}_{\mathrm{ing}}} \\
& =i A_{\tilde{\varphi}}(\boldsymbol{k}) e^{-i\left[\chi_{\mathrm{s}}(\boldsymbol{k})+\chi_{\mathrm{a}}(\boldsymbol{k})-P_{\tilde{\varphi}}(k)\right]} E(\boldsymbol{k}) .
\end{aligned}
$$

Since $\varphi(\boldsymbol{r})$ is a real function we have $P_{\tilde{\varphi}}(\boldsymbol{k})=-P_{\tilde{\varphi}}(-\boldsymbol{k})$ and hence

$$
P_{\tilde{\varphi}}(\boldsymbol{k}) \bmod 2 \pi+P_{\tilde{\varphi}}(-\boldsymbol{k}) \bmod 2 \pi=2 \pi n, n \in \mathbb{N} .
$$

Consequently,

$$
\chi_{\mathrm{s}}(\boldsymbol{k})=-\frac{1}{2}\left[\tilde{\phi}_{\mathrm{img}}(\boldsymbol{k})+\tilde{\phi}_{\mathrm{img}}(-\boldsymbol{k})\right]+\frac{\pi}{2}+\pi n .
$$

Following a similar line of reasoning and assuming a centrosymmetric object, i.e., $P_{\tilde{\varphi}}(\boldsymbol{k}) \in\{0, \pi\}$, we have

$$
\chi_{\mathrm{a}}(\boldsymbol{k})=-\frac{1}{2}\left[\tilde{\phi}_{\mathrm{img}}(\boldsymbol{k})-\tilde{\phi}_{\mathrm{img}}(-\boldsymbol{k})\right]+\pi n .
$$

Here the $\pi n$ stems from the possible $\pi$ phases of the real centrosymmetric object.

\section{APPENDIX B: OFF-AXIS HOLOGRAPHIC RECONSTRUCTION AND COMPARISON TO HRTEM}

By utilizing off-axis electron holography the phase of the electron wave can be reconstructed; to that end one part 
of the electron wave, which has interacted with the sample $\left[\Psi_{\text {img }}(\boldsymbol{r})\right]$, is superimposed with a plane reference wave $\left[\Psi_{\text {ref }}(\boldsymbol{r})\right]$ by means of an electrostatic Möllenstedt biprism [72] [Fig. 1(a)]. The wavefronts of these waves are tilted towards each other with an inclination angle $\boldsymbol{\beta}=\frac{\boldsymbol{k}_{\boldsymbol{c}}}{k_{0}}$. Denoting the image wave $\Psi_{\text {img }}(\boldsymbol{r})=A_{\text {img }}(\boldsymbol{r}) e^{i \frac{\boldsymbol{k}_{c}}{2} \boldsymbol{r}+i \varphi_{\text {img }}(\boldsymbol{r})}$ and the reference wave $\Psi_{\text {ref }}(\boldsymbol{r})=A_{\text {ref }} e^{-i \frac{k_{c}}{2} \boldsymbol{r}}$, the detected hologram intensity reads

$$
\begin{aligned}
I_{\mathrm{hol}}(\mathbf{r})= & \left|\Psi_{\mathrm{img}}(\boldsymbol{r})+\Psi_{\mathrm{ref}}\right|^{2} \\
= & A_{\mathrm{ref}}^{2}+A_{\mathrm{img}}^{2}(\boldsymbol{r}) \\
& +2 \mu\left(\boldsymbol{k}_{c}\right) A_{\mathrm{ref}} A_{\mathrm{img}}(\boldsymbol{r}) \cos \left[\boldsymbol{k}_{c} \boldsymbol{r}+\varphi_{\mathrm{img}}(\boldsymbol{r})\right] .
\end{aligned}
$$

Starting from this interference pattern, the electron wave in amplitude and phase is reconstructed using Fourier methods. Accordingly, the hologram intensity $I_{\text {hol }}(\boldsymbol{r})$ is Fourier transformed:

$$
\begin{aligned}
\mathcal{F}\left[I_{\text {hol }}(\mathbf{r})\right]= & \underbrace{\mathcal{F}\left\{\left[A_{\mathrm{ref}}^{2}+A_{\mathrm{img}}^{2}(\boldsymbol{r})\right] * \delta(\boldsymbol{k})\right\}}_{\text {centerband } I_{\mathrm{CB}}(\boldsymbol{k})} \\
& +\underbrace{\mathrm{A}_{\mathrm{ref}} \mathcal{F}\left[A_{\mathrm{img}}(\boldsymbol{r}) e^{-i \varphi_{\mathrm{img}}(\boldsymbol{r})}\right] * \delta\left(\boldsymbol{k}-\boldsymbol{k}_{c}\right)}_{\text {sideband " }-1^{\prime \prime} I_{\mathrm{SB}-1}(\boldsymbol{k})} \\
& +\underbrace{A_{\mathrm{ref}} \mathcal{F}\left[A_{\mathrm{img}}(\boldsymbol{r}) e^{+i \varphi_{\mathrm{img}}(\boldsymbol{r})}\right] * \delta\left(\boldsymbol{k}+\boldsymbol{k}_{c}\right)}_{\text {sideband "+1" }{ }^{\prime \prime} I_{\mathrm{SB}+1}(\boldsymbol{k})},
\end{aligned}
$$

separating the centerband and sideband in reciprocal space. The amplitude $\left[A_{\text {img }}(\boldsymbol{r})\right]$ and phase $\left[\varphi_{\text {img }}(\boldsymbol{r})\right]$ of the electron wave as well as the conventional centerband intensity can now be reconstructed numerically by masking and recentering them individually before transforming them back to position space.

Beside analyzing the reconstructed wave individually (see main text), it is also possible to compare the reconstructed phase analysis to conventional HRTEM analysis based on the normalized holographic centerband excluding the reference intensity $I_{\mathrm{CB}}=I_{\text {HRTEM }}-1$ [see Eq. (B2) and Fig. 6]. Accordingly, the centerband carries the same information as an HRTEM image, with similar noise properties. Most notably it is also affected by residual aberrations (e.g., visible as distortions of atomic columns, $\mathrm{B}$ and $\mathrm{N}$ not distinguishable) that drift during the time between correction and acquisition of the hologram series due to unavoidable instrumental instabilities. Contrary to the holographic autocorrection scheme discussed in the main text, there is no way to correct those residual aberrations in an a posteriori fashion in HRTEM images, because the phase information is lost. A further notable difference to the holographic analysis is the missing low-spatial-frequency band [see Fig. 1(b)], which, e.g., renders the steps between the different layers barely detectable (see Fig. 6). The holographic approach in principle also allows maximal contrast transfer up to the information limit in the high-spatial-frequency limit. Currently, however, high-spatial frequencies in the holograms are additionally damped by the limited modulation transfer of typical detectors, which is expected to improve considerably with the advent of the latest generation of direct detection cameras. Altogether these features allow a quantitative analysis in terms of local (atomic) potentials from holographic

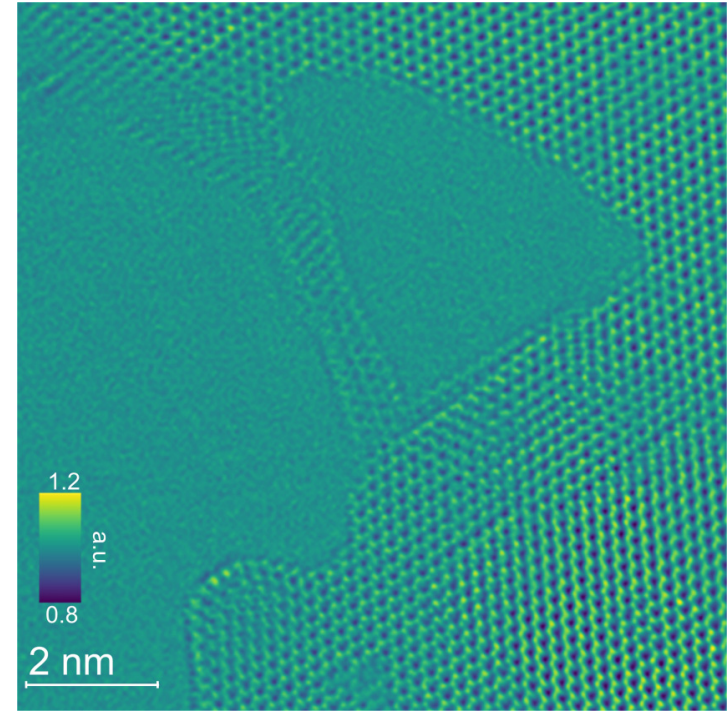

FIG. 6. Normalized holographic centerband intensity from the same hologram as used for high-resolution potential analysis (Fig. 5).

reconstructions only, whereas HRTEM analysis mainly focuses on (high-resolution) structural analysis of 2DMs.

\section{APPENDIX C: NOISE TRANSFER}

In the derivation of the SNR for off-axis holography and conventional phase contrast HRTEM we used the generalized Lenz model (uncorrelated shot and detector noise, commensurable sinc sideband mask, noise small compared to total intensity), which gives good agreement with the observed phase noise, in particular under weak contrast conditions as present in the WPO [51]. Moreover, we assumed that the noise characteristics (e.g., variance) do not depend significantly on the position on the detector, which is again a good approximation for weakly scattering objects. Using these approximations the variance of the reconstructed phase reads

$$
\sigma_{\varphi}^{2}=\frac{1}{I \mu_{\mathrm{c}}\left(\boldsymbol{k}_{\mathrm{c}}\right)^{2} \mathrm{DQE}\left(\boldsymbol{k}_{\mathrm{c}}\right)} .
$$

Here, the DQE denotes a 2D generalization of detection quantum efficiency defined as

$$
\operatorname{DQE}\left(\boldsymbol{k}_{\mathrm{c}}\right)=\frac{\operatorname{MTF}^{2}\left(\boldsymbol{k}_{\mathrm{c}}\right)}{\operatorname{NPS}\left(\boldsymbol{k}_{\mathrm{c}}\right)},
$$

with the MTF denoting the modulation transfer and NPS denoting the (normalized) white-noise power spectrum of the detector. The phase SNR then reads

$$
\mathrm{SNR}_{\mathrm{hol}}=\frac{\varphi_{\mathrm{obj}}}{\sigma_{\varphi}}=\sqrt{\frac{E^{2} \varphi_{\mathrm{obj}}^{2} I \mu_{\mathrm{c}}\left(\boldsymbol{k}_{\mathrm{c}}\right)^{2}}{\operatorname{DQE}\left(\boldsymbol{k}_{\mathrm{c}}\right)}} .
$$

The noise analysis for conventional weak phase contrast HRTEM starts with the shot noise amplified by the detector:

$$
\sigma_{\mathrm{I}}^{2}=I \operatorname{NPS}(0)
$$


from which the SNR is readily derived inserting the relation between $I$ and the phase (PCTF):

$$
\mathrm{SNR}_{\mathrm{conv}}=\sqrt{\frac{I^{2} \mathrm{PCTF}^{2} \varphi_{\mathrm{obj}}^{2}}{I \mathrm{DQE}(0)}} .
$$

We finally arrive at the ratio between both as noted in the main text:

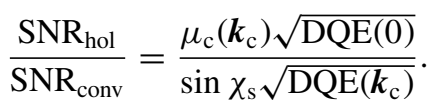

\section{APPENDIX D: MEAN INNER POTENTIAL}

Establishing a well-defined relationship between averaged projected potential or projected mean inner potential of a 2DM and the charge distribution holds some pitfalls in the infinite crystal limit [73]. Indeed, a mean inner potential is not well defined in this case and depends on fixing boundary conditions or a reference. To circumvent this problem, $a b$ initio calculations of MIPs of bulk crystals have been carried out for slab geometries, containing a sufficiently large vacuum region fixing the reference. In case of a finite crystal (as observed experimentally), we may start off with dividing the 2DM domain into "atomic" cells:

$$
V(\boldsymbol{r})=\sum V_{\mathrm{at}}(\boldsymbol{r}),
$$

which shall contain one atom each but are not further specified at this stage. The projected average of the potential over a certain area $A$, i.e.,

$$
\frac{1}{A} \int_{A} V(\boldsymbol{r}) d^{3} r=\frac{1}{A} \int \sum V_{\mathrm{at}}(\boldsymbol{r}) d^{3} r,
$$

can now be computed as a sum of the atomic contributions, which are contained within the area. This is most conveniently done in Fourier space employing the Poisson equation

$$
k^{2} \tilde{V}_{\mathrm{at}}(\boldsymbol{k})=\frac{\tilde{\rho}_{\mathrm{at}}(\boldsymbol{k})}{\varepsilon_{0}} .
$$

The atomic averaged potential now corresponds to the value at zero spatial frequency:

$$
\tilde{V}_{\mathrm{at}}(k=0)=\lim _{k \rightarrow 0} \frac{\tilde{\rho}_{\mathrm{at}}\left(k, \varphi_{k}, \theta_{k}\right)}{\varepsilon_{0} k^{2}},
$$

which is not determined in the required limit as both nominator and denominator tend to zero. To solve that expression we may apply l'Hôpital's rule to the average over the full solid angle of the previous expression (to remove the $\varphi$ and $\theta$ dependency):

$$
\begin{aligned}
& \tilde{V}_{\mathrm{at}}(k=0) \\
&=\lim _{k \rightarrow 0} \partial_{k}^{2} \frac{\left\langle\tilde{\rho}_{\mathrm{at}}(k)\right\rangle_{\varphi_{k}, \theta_{k}}}{2 \varepsilon_{0}} \\
&=\lim _{k \rightarrow 0} \frac{1}{2 \varepsilon_{0}} \partial_{k}^{2} \int d^{3} r \rho_{\mathrm{at}}(\boldsymbol{r}) \\
& \quad \times\left\langle e^{\left.i k r\left(\sin \theta_{k} \sin \theta_{r} \cos \left(\varphi_{k}-\varphi_{r}\right)+\cos \theta_{k} \cos \theta_{r}\right)\right\rangle}\right. \\
&=-\frac{1}{2 \varepsilon_{0}} \int d r^{3} r^{2} \rho_{\mathrm{at}}(\boldsymbol{r})
\end{aligned}
$$

$$
\begin{aligned}
& \times\left\langle\left[\sin \theta_{k} \sin \theta_{r} \cos \left(\varphi_{k}-\varphi_{r}\right)+\cos \theta_{k} \cos \theta_{r}\right]^{2}\right\rangle \\
= & -\frac{1}{2 \varepsilon_{0}} \int d r^{3} r^{2} \rho_{\mathrm{at}}(\boldsymbol{r}) \\
& \times\left\langle\sin ^{2} \theta_{k} \sin ^{2} \theta_{r} \cos ^{2}\left(\varphi_{k}-\varphi_{r}\right)+\cos ^{2} \theta_{k} \cos ^{2} \theta_{r}\right\rangle \\
= & -\frac{2 \pi}{3 \varepsilon_{0}} \int d r r^{2} \rho_{\mathrm{at}}(\boldsymbol{r}) .
\end{aligned}
$$

Note that we had to apply l'Hôpital's rule twice, which requires both the zeroth and any first moment with respect to $r$ to vanish. Consequently, the "atomic" cells, used for partitioning the 2D domain, have to be charge neutral and dipole-moment free. That condition restricts the allowed shapes and positions of the initially undefined atomic patch choice. Noting that dipole-free atomic cells are centered closely to the atomic positions in $h$-BN, we have that the averaged potential is a measure for the size of the electron cloud around the atoms.

\section{APPENDIX E: DFT}

Density-functional [74] band-structure calculations using the all-electron full-potential local-orbital (FPLO-18) [67] calculation scheme were employed to obtain the electronic properties (e.g., electron density and potential) of single to five layer $h$-BN and graphene for reference. The calculations were scalar relativistic [75] and used the generalized gradient approximation of the exchange-correlation functional due to Perdew-Burke-Ernzerhof [76]. The in-plane structural parameter $a=2.505 \AA$ and the distance between the layers $d=3.324 \AA$ of $h$-BN were taken from literature [77] (and agree well with our experimental findings).

\section{APPENDIX F: PCA ANALYSIS}

Principal component analysis allows one to find the optimal (with respect to the Euclidean distance) linear decomposition of a statistically varying signal into a truncated basis. It is a well-established method in advanced statistical analysis and machine learning [78], provided that a sufficiently large statistical set of signals can be collected. In TEM it finds application in the analysis of electron-energy-loss spectroscopy and energy dispersive $\mathrm{x}$-ray data amongst others $[61,79,80]$. Here we apply it to the autocorrected phase dataset decomposed into patches containing one honeycomb of the 2DM structure. Due to the high structural order of $2 \mathrm{DMs}$ these patches contain a finite number of different species, namely, one to four layer "bulk" honeycombs and corresponding edges/steps. Because several thousand honeycombs are contained in one hologram, the patches form a sufficiently large statistical basis, except some particular edge states, which were too sparse to come out in the PCA. In the PCA analysis we closely followed the steps laid out in Ref. [61].

(1) Identify patches by locating the honeycomb minima. In an iterative procedure the patch origin is refined by aligning patches with their center of mass. We also subtracted the average of each patch (which amounts to removing the first principal component).

(2) Perform PCA of the data matrix, the rows of which correspond to the different patches and the columns of which represent the interlaced spatial coordinates of the patches. 
(3) Truncate the decomposition by removing all components beyond a kink in the scree plot (showing the magnitude-ordered principal components). This filter is referred to as truncated PCA in literature.
(4) Compute the difference between original and truncated data, confirming the Gaussian noise nature of the remainder [see Fig. 3(c) in the main text].

(5) Replace the patches in the original image with the PCA truncated patches [Fig. 3(b)].
[1] K. S. Novoselov, A. K. Geim, S. V. Morozov, D. Jiang, Y. Zhang, S. V. Dubonos, I. V. Grigorieva, and A. A. Firsov, Electric field effect in atomically thin carbon films, Science 306, 666 (2004).

[2] A. K. Geim and K. S. Novoselov, The rise of graphene, Nat. Mater. 6, 183 (2007).

[3] Q. H. Wang, K. Kalantar-Zadeh, A. Kis, J. N. Coleman, and M. S. Strano, Electronics and optoelectronics of two-dimensional transition metal dichalcogenides, Nat. Nanotechnol. 7, 699 (2012).

[4] S. Z. Butler, S. M. Hollen, L. Cao, Y. Cui, J. A. Gupta, H. R. Gutiérrez, T. F. Heinz, S. Sae Hong, J. Huang, A. F. Ismach, E. Johnston-Halperin, M. Kuno, V. V. Plashnitsa, R. D. Robinson, R. S. Ruoff, S. Salahuddin, J. Shan, L. Shi, M. G. Spencer, M. Terrones, W. Windl, and J. E. Goldberger, Progress, challenges, and opportunities in two-dimensional materials beyond graphene, ACS Nano 7, 2898 (2013).

[5] P. Miró, M. Audiffred, and T. Heine, An atlas of two-dimensional materials, Chem. Soc. Rev. 43, 6537 (2014).

[6] A. J. Mannix, B. Kiraly, M. C. Hersam, and N. P. Guisinger, Synthesis and chemistry of elemental $2 d$ materials, Nat. Rev. Chem. 1, 0014 (2017).

[7] O. Lopez-Sanchez, D. Lembke, M. Kayci, A. Radenovic, and A. Kis, Ultrasensitive photodetectors based on monolayer $\mathrm{MoS}_{2}$, graphene, Nat. Nanotechnol. 8, 497 (2013).

[8] R. Peierls, Quelques propriétés typiques des corps solides, Ann. Inst. Henri Poincare 5, 177 (1935).

[9] J. C. Meyer, A. K. Geim, M. I. Katsnelson, K. S. Novoselov, T. J. Booth, and S. Roth, The structure of suspended graphene sheets, Nature (London) 446, 60 (2007).

[10] S. Deng and V. Berry, Wrinkled, rippled and crumpled graphene: An overview of formation mechanism, electronic properties, and applications, Mater. Today 19, 197 (2016).

[11] Z. Lin, B. R. Carvalho, E. Kahn, R. Lv, R. Rao, H. Terrones, M. A Pimenta, and M. Terrones, Defect engineering of twodimensional transition metal dichalcogenides, 2D Mater. 3, 022002 (2016).

[12] M. Linck, P. Hartel, S. Uhlemann, F. Kahl, H. Müller, J. Zach, M. Haider, M. Niestadt, M. Bischoff, J. Biskupek, Z. Lee, T. Lehnert, F. Börrnert, H. Rose, and U. Kaiser, Chromatic Aberration Correction for Atomic Resolution TEM Imaging from 20 to $80 \mathrm{kV}$, Phys. Rev. Lett. 117, 076101 (2016).

[13] H.-P. Komsa, J. Kotakoski, S. Kurasch, O. Lehtinen, U. Kaiser, and A. V. Krasheninnikov, Two-Dimensional Transition Metal Dichalcogenides Under Electron Irradiation: Defect Production and Doping, Phys. Rev. Lett. 109, 035503 (2012).

[14] T. Lehnert, O. Lehtinen, G. Algara-Siller, and U. Kaiser, Electron radiation damage mechanisms in $2 \mathrm{D} \mathrm{MoSe}_{2}$, Appl. Phys. Lett. 110, 033106 (2017).
[15] D. Zhang, Y. Zhu, L. Liu, X. Ying, C.-En Hsiung, R. Sougrat, $\mathrm{K}$. Li, and Y. Han, Atomic-resolution transmission electron microscopy of electron beam-sensitive crystalline materials, Science 359, 675 (2018).

[16] J. C. Meyer, F. Eder, S. Kurasch, V. Skakalova, J. Kotakoski, H. Jin Park, S. Roth, A. Chuvilin, S. Eyhusen, G. Benner, A. V. Krasheninnikov, and U. Kaiser, Accurate Measurement of Electron Beam Induced Displacement Cross Sections for Single-Layer Graphene, Phys. Rev. Lett. 108, 196102 (2012).

[17] J. Kotakoski, C. H. Jin, O. Lehtinen, K. Suenaga, and A. V. Krasheninnikov, Electron knock-on damage in hexagonal boron nitride monolayers, Phys. Rev. B 82, 113404 (2010).

[18] T. Susi, J. C. Meyer, and J. Kotakoski, Quantifying transmission electron microscopy irradiation effects using two-dimensional materials, Nat. Rev. Phys. 1, 397 (2019).

[19] V. H. Boersch, Über die kontraste von atomen in electronenmikroskop, Z. Naturforsch. A 2, 615 (1947).

[20] K. Kanaya, H. Kawakatsu, K. Itō, and H. Yotsumoto, Experiment on the electron phase microscope, J. Appl. Phys. 29, 1046 (1958).

[21] R. Danev and K. Nagayama, Transmission electron microscopy with zernike phase plate, Ultramicroscopy 88, 243 (2001).

[22] R. Danev, B. Buijsse, M. Khoshouei, J. M. Plitzko, and W. Baumeister, Volta potential phase plate for in-focus phase contrast transmission electron microscopy, Proc. Natl. Acad. Sci. USA 111, 15635 (2014).

[23] T. Matsumoto and A. Tonomura, The phase constancy of electron waves traveling through Boersch's electrostatic phase plate, Ultramicroscopy 63, 5 (1996).

[24] E. Majorovits, B. Barton, K. Schultheiss, F. Perez-Willard, D. Gerthsen, and R. R. Schröder, Optimizing phase contrast in transmission electron microscopy with an electrostatic (Boersch) phase plate, Ultramicroscopy 107, 213 (2007).

[25] R. Cambie, K. H. Downing, D. Typke, R. M. Glaeser, and J. Jin, Design of a microfabricated, two-electrode phase-contrast element suitable for electron microscopy, Ultramicroscopy $\mathbf{1 0 7}$, 329 (2007).

[26] K. Schultheiß, F. Pérez-Willard, B. Barton, D. Gerthsen, and R. R. Schröder, Fabrication of a Boersch phase plate for phase contrast imaging in a transmission electron microscope, Rev. Sci. Instrum. 77, 033701 (2006).

[27] K. Nagayama and R. Danev, Phase contrast electron microscopy: development of thin-film phase plates and biological applications, Phil. Trans. R. Soc. B 363, 2153 (2008).

[28] O. Schwartz, J. J. Axelrod, S. L. Campbell, C. Turnbaugh, R. M Glaeser, and H. Müller, Laser phase plate for transmission electron microscopy, Nat. Methods 16, 1016 (2019).

[29] P. Simon, H. Lichte, R. Wahl, M. Mertig, and W. Pompe, Electron holography of non-stained bacterial surface layer proteins, Biochimica et Biophysica Acta (BBA): Biomembranes 1663 , 178 (2004). 
[30] P. Simon, H. Lichte, P. Formánek, M. Lehmann, R. Huhle, W. Carrillo-Cabrera, A. Harscher, and H. Ehrlich, Electron holography of biological samples, Micron 39, 229 (2008).

[31] L. Ortolani, F. Houdellier, M. Monthioux, E. Snoeck, and V. Morandi, Surface electrostatic potentials in carbon nanotubes and graphene membranes investigated with electron holography, Carbon 49, 1423 (2011).

[32] D. Cooper, C.-T. Pan, and L. Sarah Haigh, Atomic resolution electrostatic potential mapping of graphene sheets by off-axis electron holography, J. Appl. Phys. 115, 233709 (2014).

[33] A. H. Tavabi, F. Winkler, Y.-C. Lin, K. Suenaga, E. Yucelen, R. E. Dunin-Borkowski, and B. E. Kardynal, Aberration corrected off-axis electron holography of layered transition metal dichalcogenides, Microsc. Microanal. 21, 1399 (2015).

[34] S. Borghardt, F. Winkler, Z. Zanolli, M. J. Verstraete, J. Barthel, A. H. Tavabi, R. E. Dunin-Borkowski, and B. E. Kardynal, Quantitative Agreement between Electron-Optical Phase Images of $\mathrm{wse}_{2}$ and Simulations Based on Electrostatic Potentials that Include Bonding Effects, Phys. Rev. Lett. 118, 086101 (2017).

[35] F. Winkler, A. H. Tavabi, J. Barthel, M. Duchamp, E. Yucelen, S. Borghardt, B. E. Kardynal, and R. E. Dunin-Borkowski, Quantitative measurement of mean inner potential and specimen thickness from high-resolution off-axis electron holograms of ultra-thin layered wse2, Ultramicroscopy 178, 38 (2017).

[36] F. Winkler, J. Barthel, A. H. Tavabi, S. Borghardt, B. E. Kardynal, and R. E. Dunin-Borkowski, Absolute Scale Quantitative Off-Axis Electron Holography at Atomic Resolution, Phys. Rev. Lett. 120, 156101 (2018).

[37] J. Barthel and A. Thust, Aberration measurement in HRTEM: Implementation and diagnostic use of numerical procedures for the highly precise recognition of diffractogram patterns, Ultramicroscopy 111, 27 (2010).

[38] V. Boureau, B. Sklenard, R. McLeod, D. Ovchinnikov, D. Dumcenco, A. Kis, and D. Cooper, Quantitative mapping of the charge density in a monolayer of $\mathrm{MoS}_{2}$ at atomic resolution by off-axis electron holography, ACS Nano 14, 524 (2020).

[39] T. Niermann and M. Lehmann, Averaging scheme for atomic resolution off-axis electron holograms, Micron 63, 28 (2014).

[40] Q. Fu, H. Lichte, and E. Völkl, Corrections of Aberrations of an Electron Microscope by Means of Electron Holography, Phys. Rev. Lett. 67, 2319 (1991).

[41] D. Gabor, A new microscopic principle, Nature (London) 161, 777 (1948).

[42] M. Lentzen, B. Jahnen, C. L. Jia, A. Thust, K. Tillmann, and K. Urban, High-resolution imaging with an aberration-corrected transmission electron microscope, Ultramicroscopy 92, 233 (2002).

[43] M. Haider, P. Hartel, H. Müller, S. Uhlemann, and J. Zach, Information transfer in a TEM corrected for spherical and chromatic aberration, Microsc. Microanal. 16, 393 (2010).

[44] S. Uhlemann, H. Müller, P. Hartel, J. Zach, and M. Haider, Thermal Magnetic Field Noise Limits Resolution in Transmission Electron Microscopy, Phys. Rev. Lett. 111, 046101 (2013).

[45] N. Alem, R. Erni, C. Kisielowski, M. D. Rossell, W. Gannett, and A. Zettl, Atomically thin hexagonal boron nitride probed by ultrahigh-resolution transmission electron microscopy, Phys. Rev. B 80, 155425 (2009).

[46] W. M. J. Coene, A. Thust, M. Op de Beeck, and D. Van Dyck, Maximum-likelihood method for focus-variation image recon- struction in high resolution transmission electron microscopy, Ultramicroscopy 64, 109 (1996).

[47] A. I. Kirkland, W. O. Saxton, K.-L. Chau, K. Tsuno, and M. Kawasaki, Super-resolution by aperture synthesis: tilt series reconstruction in CTEM, Ultramicroscopy 57, 355 (1995).

[48] A. M. Maiden and J. M. Rodenburg, An improved ptychographical phase retrieval algorithm for diffractive imaging, Ultramicroscopy 109, 1256 (2009).

[49] M. Lehmann and H. Lichte, Tutorial on off-axis electron holography, Microsc. Microanal. 8, 447 (2002).

[50] A. Lubk, F. Rder, T. Niermann, C. Gatel, S. Joulie, F. Houdellier, C. Magen, and M. J. Hytch, A new linear transfer theory and characterization method for image detectors. Part ii: Experiment, Ultramicroscopy 115, 78 (2012).

[51] F. Röder, A. Lubk, D. Wolf, and T. Niermann, Noise estimation for off-axis electron holography, Ultramicroscopy 144, 32 (2014).

[52] R. S. Ruskin, Z. Yu, and N. Grigorieff, Quantitative characterization of electron detectors for transmission electron microscopy, J. Struct. Biol. 184, 385 (2013).

[53] G. McMullan, A. R. Faruqi, D. Clare, and R. Henderson, Comparison of optimal performance at $300 \mathrm{keV}$ of three direct electron detectors for use in low dose electron microscopy, Ultramicroscopy 147, 156 (2014).

[54] F. Zemlin and P. Schiske, Measurement of the phase contrast transfer function and the cross-correlation peak using young interference fringes, Ultramicroscopy 5, 139 (1980).

[55] M. Lehmann, Determination and correction of the coherent wave aberration from a single off-axis electron hologram by means of a genetic algorithm, Ultramicroscopy 85, 165 (2000).

[56] K. Ishizuka, T. Tanji, A. Tonomura, T. Ohno, and Y. Murayama, Aberration correction using off-axis holography i. aberration assessment, Ultramicroscopy 53, 361 (1994).

[57] M. Linck, Off-axis Holografie im aberrationskorrigierten Transmissionselektronenmikroskop, Ph.D. thesis, Technische Universität Dresden, 2010, https://nbn-resolving.org/urn:nbn:de: bsz:14-qucosa-38972.

[58] G. Cassabois, P. Valvin, and B. Gil, Hexagonal boron nitride is an indirect bandgap semiconductor, Nat. Photonics 10, 262 (2016).

[59] J. Wang, F. Ma, and M. Sun, Graphene, hexagonal boron nitride, and their heterostructures: properties and applications, RSC Advances 7, 16801 (2017).

[60] M. Linck, D. Wolf, A. Lubk, and F. Röder, Improved object wave reconstruction in off axis holography, in Proceedings of MC2009, Vol. 1: Instrumentation and Methodology, edited by G. Kothleitner and M. Leisch (Facultas (Vienna), 2009), pp. 31-32.

[61] P. Potapov and A. Lubk, Optimal principal component analysis of stem XEDS spectrum images, Adv. Struct. Chem. Imaging 5, 4 (2019).

[62] N. Alem, O. V. Yazyev, C. Kisielowski, P. Denes, U. Dahmen, P. Hartel, M. Haider, M. Bischoff, B. Jiang, S. G. Louie, and A. Zettl, Probing the Out-of-Plane Distortion of Single Point Defects in Atomically thin Hexagonal Boron Nitride at the Picometer Scale, Phys. Rev. Lett. 106, 126102 (2011).

[63] N. Alem, Q. M. Ramasse, C. R. Seabourne, O. V. Yazyev, K. Erickson, M. C. Sarahan, C. Kisielowski, A. J. Scott, S. G. Louie, and A. Zettl, Subangstrom Edge Relaxations Probed by 
Electron Microscopy in Hexagonal Boron Nitride, Phys. Rev. Lett. 109, 205502 (2012)

[64] M. O'Keeffe and J. C. H. Spence, On the average Coulomb potential $\left(\Sigma_{0}\right)$ and constraints on the electron density in crystals, Acta Crystallogr. Sect. A 50, 33 (1994).

[65] J. A. Ibers, Atomic scattering amplitudes for electrons, Acta Crystallogr. 11, 178 (1958).

[66] A. Weickenmeier and H. Kohl, Computation of absorptive form factors for high-energy electron diffraction, Acta Crystallogr. Sect. A 47, 590 (1991).

[67] K. Koepernik and H. Eschrig, Full-potential nonorthogonal local-orbital minimum-basis band-structure scheme, Phys. Rev. B 59, 1743 (1999).

[68] V. Barone and J. E. Peralta, Magnetic boron nitride nanoribbons with tunable electronic properties, Nano Lett. 8, 2210 (2008).

[69] H. Zeng, C. Zhi, Z. Zhang, X. Wei, X. Wang, W. Guo, Y. Bando, and D. Golberg, White graphenes: Boron nitride nanoribbons via boron nitride nanotube unwrapping, Nano Lett. 10, 5049 (2010).

[70] A. Lopez-Bezanilla, J. Huang, H. Terrones, and B. G. Sumpter, Boron nitride nanoribbons become metallic, Nano Lett. 11, 3267 (2011).

[71] X. Fu and R. Zhang, Energetics of hexagonal boron nitride nanostructures: Edge dependence and truncation effects, Nanoscale 9, 6734 (2017).
[72] G. Möllenstedt and H. Düker, Interferenzversuch mit einem Biprisma für Elektronenwellen, Naturwissenschaften 42, 41 (1955).

[73] L. Kleinman, Comment on the average potential of a Wigner solid, Phys. Rev. B 24, 7412 (1981).

[74] P. Hohenberg and W. Kohn, Inhomogeneous electron gas, Phys. Rev. 136, B864 (1964).

[75] H. Eschrig, M. Richter, and I. Opahle, Relativistic Electronic Structure Theory, Part 2: Applications, Theoretical and Computational Chemistry (Elsevier, Amsterdam, 2004), Vol. 13, pp. 723-776.

[76] J. P. Perdew, K. Burke, and M. Ernzerhof, Generalized Gradient Approximation Made Simple, Phys. Rev. Lett. 77, 3865 (1996).

[77] W. Paszkowicz, J. B. Pelka, M. Knapp, T. Szyszko, and S. Podsiadlo, Lattice parameters and anisotropic thermal expansion of hexagonal boron nitride in the 10-297.5 K temperature range, Appl. Phys. A 75, 431 (2002).

[78] I. T. Jolliffe and J. Cadima, Principal component analysis: a review and recent developments, Philos. Trans. R. Soc. A 374, 20150202 (2016).

[79] J. M. Titchmarsh and S. Dumbill, Multivariate statistical analysis of feg-stem edx spectra, J. Microsc. 184, 195 (1996).

[80] C. M. Parish and L. N. Brewer, Multivariate statistics-based segmentation methods for quantification of x-ray spectrum images, Ultramicroscopy 110, 134 (2010). 